\title{
Folgen von Höhenfußpunktdreiecken und ihre Grenzpunkte
}

\author{
Evgeny Strekalovskiy \\ Evgeny Strekalovskiy hat 2004-06 an der Friedrich-Schiller-Universität Jena Mathe- \\ matik und Informatik studiert. Gegenwärtig setzt er sein Studium an der Rheinischen \\ Friedrich-Wilhelms-Universität Bonn fort. Er ist dreifacher Bundessieger im Bundes \\ wettbewerb Mathematik und Bundessieger im Wettbewerb Jugend forscht.
}

\section{Einleitung}

Betrachtet man irgendeine Abbildung, die jedem Dreieck der euklidischen Ebene eindeutig sein „Nachfolgedreieck“ zuordnet, so entsteht für jedes Ausgangsdreieck $\Delta_{0}$ eine Folge $\Delta_{0}, \Delta_{1}, \Delta_{2}, \ldots$ von Dreiecken. Es gibt sicherlich unzählige Möglichkeiten solche Dreiecksfolgen zu konstruieren. Eine besonders interessante Folge entsteht, wenn man als Nachfolgedreieck jeweils das aus den drei Höhenfußpunkten gebildete Höhenfußpunktdreieck nimmt. Es zeigt sich, dass diese Folge immer gegen einen „Grenzpunkt" strebt, sobald sie bloß beliebig weit fortsetzbar ist. Das Bemerkenswerte hierbei ist, dass die Lage dieses Grenzpunktes bei gleichschenkligem Ausgangsdreieck durch eine stetige aber nirgendwo differenzierbare Funktion beschrieben wird, wodurch sich die Folge von vielen anderen deutlich auszeichnet.

Diese Folge erfreute sich bereits großer Aufmerksamkeit seitens verschiedener Autoren, jedoch war fast ausschließlich das Verhalten der Innenwinkel der Folgendreiecke Gegenstand der Untersuchung, und nicht das des Grenzpunktes. Die wohl erste Beschreibung

Verbindet man in einem Dreieck $H_{0}$ die drei Höhenfußpunkte, so entsteht ein neues Dreieck $H_{1}$, und Wiederholung dieser Konstruktion liefert eine Folge $H_{0}, H_{1}, H_{2}, \ldots$ von immer kleineren Höhenfußpunktdreiecken, die gegen einen Grenzpunkt konvergieren. Diese Folge hat schon die Aufmerksamkeit vieler Mathematiker gefunden, darunter Lax und Conway. Unser Autor berichtet darüber und stellt explizite Formeln für die Folgendreiecke auf. Es stellt sich heraus, dass der Grenzpunkt eine stetige, aber nirgends differenzierbare Funktion des Ausgangsdreiecks ist. Eine besondere Rolle spielt dabei der Fall, wo im Lauf der Konstruktion ein rechtwinkliges Dreieck entsteht. 
findet sich bei Hobson [7] bereits 1891. Daraufhin untersuchte Vályi [14] die allgemeine Gestalt und Anzahl periodischer Folgen (s. Abschnitt 4) auf rein kombinatorischem Wege (s. Folge A102536 in [10]) und Tuckey [12] stellte die Entstehung der 12-Periodizität fest, wenn das Gradmaß der Anfangswinkel ganzzahlig ist. Diese Tatsache sowie einige weitere Eigenschaften streng periodischer Folgen haben des Weiteren Kingston und Synge [8] untersucht. Sie führten die Verhältnisgrößen ein, arbeiteten jedoch wie auch in [14] und [12] direkt mit den Innenwinkeln selbst, wodurch ein größerer Anteil der Resultate und Beweise eher technisch erscheint. Hierauf nahm Alexander [1] Bezug und beschrieb, wie sich der Übergang zum jeweils nächsten Folgendreieck als Shift auf einer bestimmten Kodierung der Dreiecke erweist und so jede Untersuchung der Innenwinkel auf die dieser Kodierungen zurückführbar ist, wobei die letztere bequem mit den mächtigen Mitteln der symbolischen Dynamik erfolgen kann. Möchte man Aussagen direkt über die Innenwinkel selbst und nicht auf dem Umweg über die Kodierung erhalten, bedarf es dann allerdings immer einer „Übersetzung“. Im Gegensatz dazu werden wir in der vorliegenden Arbeit die Innenwinkel von vorn herein ganz im Lichte der Binärdarstellungen der Verhältnisgrößen behandeln. Mit dieser Methode gelangt man auf einheitlichem Wege schnell, einfach und durchsichtig zu wesentlichen unmittelbaren Aussagen über die Innenwinkel selbst. In dieser Form finden sie sich nicht in früherer Literatur. So wird insbesondere die Herleitung des bemerkenswerten Satzes 4.4 ermöglicht, der wie auch der Satz 4.5 ein neues Resultat darstellt. In eine andere Richtung gingen Lax [9] und später Ungar [13], indem sie die sog. Mischungseigenschaft nachwiesen. Diese impliziert, dass „fast jedes“ Ausgangsdreieck zu einer Folge führt, in welcher man von der Größe abgesehen früher oder später Folgendreiecke nahezu jeder möglichen Gestalt antrifft.

Untersuchungen bezüglich des Grenzpunktes gibt es weitaus weniger. Eine Formel für den gleichschenkligen Fall wird in [11] und [2] hergeleitet, in der letzten Arbeit wird die erhaltene Funktion auch als nirgendwo differenzierbar herausgestellt. Die Frage nach dem Grenzpunkt kam erneut in [5] durch Conway et al. auf. Hier findet man einen Existenznachweis, das Fortsetzbarkeitskriterium 4.2 und zum ersten Mal eine auf Conway zurückgehende analytische Herleitung der expliziten Formel aus Satz 2.3 für ein allgemeines Ausgangsdreieck. Conway wies auf die durch die Stetigkeit mögliche Erweiterung der Definition des Grenzpunktes auf die abbrechenden Folgen, ließ jedoch offen, welcher Punkt genau als Grenzpunkt genommen werden soll. In der vorliegenden Arbeit präsentieren wir einen synthetischen Beweis der Formel und klären die letzte Frage vollständig. Die allgemeine Formel für die Lage des Grenzpunktes relativ zum Mittelpunkt einer Seite in Satz 2.4 und die gesamte Untersuchung zu Höchstentfernungen im Abschnitt 3 kommen in früheren Arbeiten nicht vor. Der Grenzpunkt $H_{\infty}$ eines Dreiecks ist noch nicht als ein Kimberling Center $X_{n}$ erfasst (Encyclopedia of Triangle Centers).

Die vorliegende Arbeit ist in drei Abschnitte unterteilt. Zunächst wird die Existenz des Grenzpunktes nachgewiesen, und Formeln für seine Lage werden hergeleitet. Im nächsten Abschnitt wird die Entfernung des Grenzpunktes zu bestimmten Punkten des Ausgangsdreiecks untersucht. Der letzte Teil behandelt schließlich die Folgendreiecke an sich, und zwar in Bezug auf ihre Innenwinkel. 


\section{Die Folge der Höhenfußpunktdreiecke}

Zuerst soll der Begriff „Grenzpunkt“ einer Dreiecksfolge festgelegt werden. Wir nennen einen Punkt $P$ Grenzpunkt der Dreiecksfolge $\left(\Delta A_{n} B_{n} C_{n}\right)$, falls die drei Eckpunktfolgen $\left(A_{n}\right),\left(B_{n}\right)$ und $\left(C_{n}\right)$ allesamt gegen $P$ konvergieren. Ggf. nennen wir die Dreiecksfolge konvergent. Wir führen nun die zentrale Dreiecksfolge dieser Arbeit ein.

Definition 2.1. Sei $\Delta$ ein nichtrechtwinkliges Dreieck. Das durch die Verbindung seiner drei Höhenfußpunkte entstehende Dreieck heißt das Höhenfußpunktdreieck von $\Delta$. Eine Dreiecksfolge $H_{0}, H_{1}, H_{2}, \ldots$, bei welcher $H_{k+1}$ das Höhenfußpunktdreieck von $H_{k}$ für jedes $k$ ist, heißt eine Folge von Höhenfußpunktdreiecken. Mit $A_{k+1}, B_{k+1}$ bzw. $C_{k+1}$ wird diejenige Ecke von $H_{k+1}$ bezeichnet, die in $H_{k}$ auf der der Ecke $A_{k}, B_{k}$ bzw. $C_{k}$ gegenüberliegenden Seite liegt.
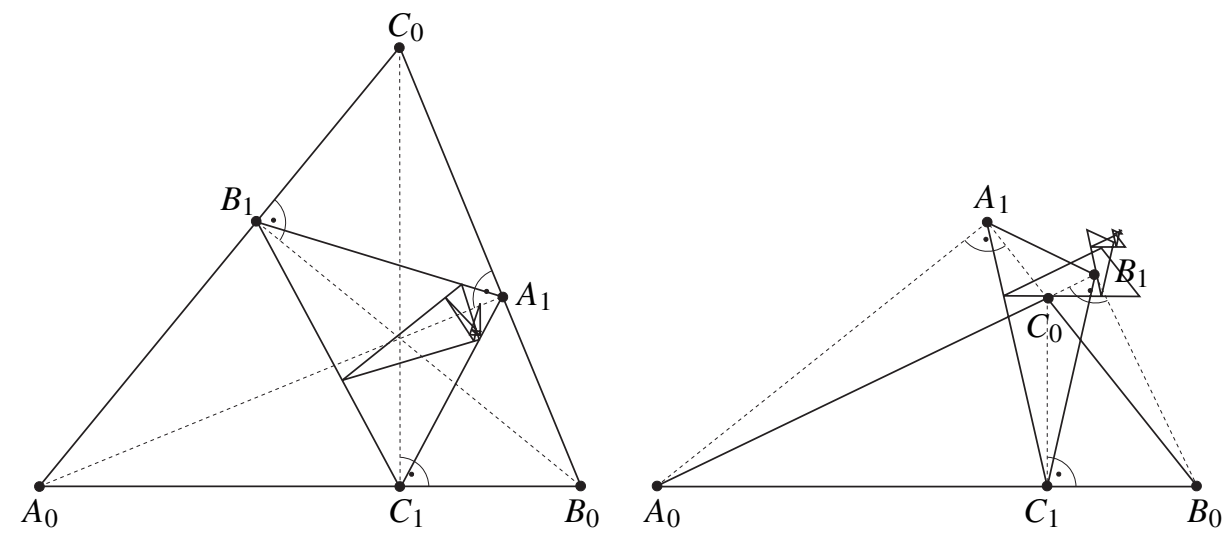

Fig. 1 Zwei Folgen von Höhenfußpunktdreiecken

Eine solche Folge bricht ggf. irgendwann mit einem rechtwinkligen Dreieck ab, da dann zwei Höhenfußpunkte zusammenfallen. Das Fortsetzbarkeitskriterium, das wir im Abschnitt 4 beweisen werden, besagt, dass dies genau dann passiert, wenn einer der Innenwinkel des Ausgangsdreiecks die Form $\frac{k}{2^{n}} \pi$ mit ganzen $k$ und $n$ hat.

Satz 2.2. Jede unendliche Folge von Höhenfußpunktdreiecken ist konvergent.

Beweis. Sei $O_{n}$ der Umkreismittelpunkt und $r_{n}$ der Umkreisradius des $n$-ten Folgendreiecks $H_{n}$. Aus der Elementargeometrie ist bekannt, dass der Umkreisradius des Höhenfußpunktdreiecks gleich der Hälfte des Umkreisradius des ursprünglichen Dreiecks ist (der Umkreis des erstgenannten Dreiecks ist der Feuerbach-Kreis des anderen), d.h. $r_{n+1}=\frac{1}{2} r_{n}$. Mit $r:=r_{0}$ folgt hieraus induktiv

$$
r_{n}=\frac{r}{2^{n}}
$$


In jedem Folgendreieck $H_{n}$ liegt der Fußpunkt der Höhe auf eine längste Seite stets auf dieser Seite, d.h. $H_{n+1}$ und $H_{n}$ und damit auch die entsprechenden Umkreise schneiden sich. Der Abstand $d\left(O_{n}, O_{n+1}\right)$ ihrer Mittelpunkte ist folglich höchstens gleich der Summe ihrer Radien, d.h.

$$
d\left(O_{n}, O_{n+1}\right) \leq r_{n}+r_{n+1}=\frac{3 r}{2^{n+1}} .
$$

Hieraus folgt leicht, dass $\left(O_{n}\right)$ eine Cauchy-Folge ist, womit $\lim _{n \rightarrow \infty} O_{n}$ existiert. Wegen $r_{n} \rightarrow 0$ ist dies auch der Grenzpunkt der drei Eckpunktfolgen.

Der Begriff des „Grenzpunktes“ kann aber auch auf die abbrechenden Folgen von Höhenfußpunktdreiecken sinnvoll erweitert werden. Falls nämlich das $n$-te Folgendreieck $H_{n}$ rechtwinklig ist, so definieren wir den Grenzpunkt dieser Folge als diejenige Ecke von $H_{n}$, bei der der rechte Winkel ist. Wir werden später sehen, dass dies eine durchaus natürliche Definition ist.

Somit besitzt also jede Folge von Höhenfußpunktdreiecken, ob abbrechend oder nicht, einen Grenzpunkt. Die folgenden zwei Sätze geben für ein gegebenes Ausgangsdreieck die genaue Lage des $n$-ten Folgendreiecks und die des Grenzpunktes relativ zum Umkreismittelpunkt sowie zu der Mitte einer Seite an. Die euklidische Ebene wird dabei mit der komplexen Zahlenebene identifiziert (abkürzend werden wir $\overrightarrow{P_{1} P_{2}}:=P_{2}-P_{1}$ für den Vektor von $P_{1}$ nach $P_{2}$ schreiben). Es seien $O$ und $r$ der Umkreismittelpunkt und -radius des Ausgangsdreiecks; $\bar{\alpha}, \bar{\beta}, \bar{\gamma}$ seien die Richtungswinkel der Vektoren vom Umkreismittelpunkt zu den drei Ecken, d.h. so dass $A=O+r e^{i \bar{\alpha}}, B=O+r e^{i \bar{\beta}}, C=O+r e^{i \bar{\gamma}}$. Die entsprechenden Größen des $n$-ten Folgendreiecks $H_{n}$ werden mit dem Index $n$ versehen. Der Grenzpunkt wird mit $H_{\infty}$ bezeichnet. Schließlich setzen wir $\omega:=\frac{\bar{\alpha}+\bar{\beta}+\bar{\gamma}}{3}$ und

$$
\varphi_{n}:=\omega+(-2)^{n}(\bar{\alpha}-\omega), \quad \chi_{n}:=\omega+(-2)^{n}(\bar{\beta}-\omega), \quad \psi_{n}:=\omega+(-2)^{n}(\bar{\gamma}-\omega) .
$$

Satz 2.3. Es gilt

$$
H_{\infty}=O+r \sum_{n=0}^{\infty} \frac{(-1)^{n}}{2^{n+1}}\left(e^{i \varphi_{n}}+e^{i \chi_{n}}+e^{i \psi_{n}}\right)
$$

Falls $H_{n}$ existiert, ergibt der Abbruch der Reihe nach $n$ Gliedern $O_{n}$, und es gilt

$$
A_{n}=O_{n}+r \frac{(-1)^{n}}{2^{n}} e^{i \varphi_{n}}, \quad B_{n}=O_{n}+r \frac{(-1)^{n}}{2^{n}} e^{i \chi_{n}}, \quad C_{n}=O_{n}+r \frac{(-1)^{n}}{2^{n}} e^{i \psi_{n}}
$$

Beweis. Wir zeigen zuerst die zweite Aussage des Satzes über die Lage der Folgendreiecke. Die Dreiecke $H_{0}$ bis $H_{m}$ mögen existieren. Aus der elementaren Geometrie ist bekannt, dass die drei Höhenfußpunkte von $H_{n}$, d.h. die Ecken von $H_{n+1}$, auf dem Feuerbach-Kreis $k$ liegen (zusammen mit den drei Seitenmittelpunkten), dessen Mittelpunkt $F$ der Mittelpunkt der Strecke $O_{n} P$ ist, wenn $P$ den Höhenschnittpunkt bezeichnet; der Radius von $k$ ist außerdem $r^{\prime}=\frac{1}{2} r_{n}$. Der Satz von Hamilton [3] besagt aber 
$\overrightarrow{O_{n} P}=\overrightarrow{O_{n} A_{n}}+\overrightarrow{O_{n} B_{n}}+\overrightarrow{O_{n} C_{n}}$, so dass zusammen folgt

$$
O_{n+1}=F=O_{n}+\frac{\overrightarrow{O_{n} A_{n}}+\overrightarrow{O_{n} B_{n}}+\overrightarrow{O_{n} C_{n}}}{2} .
$$

Mit den Bezeichnungen in der Skizze (für den Fall eines spitzen Dreiecks $H_{n}$, der andere Fall geht analog) ist $\overline{O_{n} S}=\overline{M_{c} C_{n+1}}=2 \overline{R C_{n+1}}$ und $\overline{O_{n} C_{n}}=r_{n}=2 r^{\prime}=2 \overline{C_{n+1} F}$, d.h. die beiden Dreiecke $\Delta O_{n} S C_{n}$ und $\Delta C_{n+1} R F$ sind ähnlich, also $\angle R C_{n+1} F=\angle S O_{n} C_{n}$.

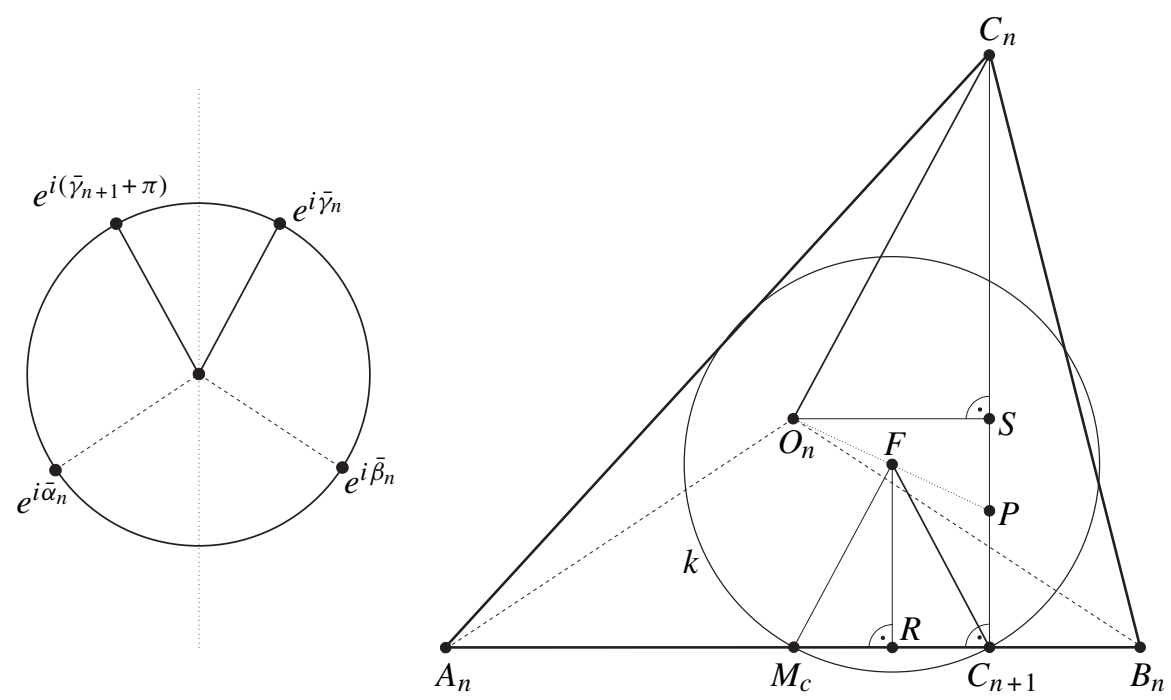

Fig. 2 Skizze zum Beweis von Satz 2.3

Der Vektor $\overrightarrow{C_{n+1} F}$ geht also aus dem Vektor $\overrightarrow{O_{n} C_{n}}$ durch Spiegelung an einer Senkrechten zu $A_{n} B_{n}$ (und Stauchung) hervor. Die Differenzen zwischen den Richtungswinkeln von $\overrightarrow{O_{n} C_{n}}$ und $\overrightarrow{O_{n} B_{n}}$ bzw. $\overrightarrow{O_{n} A_{n}}$ und $\overrightarrow{C_{n+1} F}$ müssen damit modulo $2 \pi$ übereinstimmen (siehe Fig. 2 links), d.h.

$$
\bar{\gamma}_{n}-\bar{\beta}_{n}=\bar{\alpha}_{n}-\left(\bar{\gamma}_{n+1}+\pi\right) \quad \text { bzw. } \quad \bar{\gamma}_{n+1}=\bar{\alpha}_{n}+\bar{\beta}_{n}-\bar{\gamma}_{n}+\pi .
$$

Analoge Rekursionsgleichungen ergeben sich auch für $\bar{\alpha}_{n+1}$ und $\bar{\beta}_{n+1}$. Von diesen ausgehend beweist man leicht mit vollständiger Induktion

$$
\begin{gathered}
\bar{\alpha}_{n}=\omega+(-2)^{n}(\bar{\alpha}-\omega)+n \pi, \quad \bar{\beta}_{n}=\omega+(-2)^{n}(\bar{\beta}-\omega)+n \pi, \\
\bar{\gamma}_{n}=\omega+(-2)^{n}(\bar{\gamma}-\omega)+n \pi,
\end{gathered}
$$

d.h. $\bar{\alpha}_{n}=\varphi_{n}+n \pi$ und mit (2.1) also $A_{n}=O_{n}+r_{n} e^{i \bar{\alpha}_{n}}=O_{n}+r \frac{(-1)^{n}}{2^{n}} e^{i \varphi_{n}}$ usw. Mit (2.2) liefert das insgesamt die zweite Aussage des Satzes.

Im Fall einer unendlichen Folge haben wir im Beweis des Existenzsatzes gesehen, dass die Umkreismittelpunkte $O_{n}$ gegen $H_{\infty}$ konvergieren. Nach dem soeben Gezeigten konvergieren sie aber auch gegen die im Satz angegebene Reihe. 
Sei nun ein Folgendreieck $H_{m}$ rechtwinklig. Der rechte Winkel liege o.B.d.A. bei $C_{m}$. Zu zeigen ist, dass die Reihe des Satzes gegen $C_{m}$ konvergiert. Nach dem Satz von Thales verläuft die Seite $A_{m} B_{m}$ durch den Umkreismittelpunkt, womit die Vektoren $\overrightarrow{O_{m} A_{m}}$ und $\overrightarrow{O_{m} B_{m}}$ offenbar entgegengerichtet sind, d.h. $\bar{\alpha}_{m}=\bar{\beta}_{m}+\pi$ (modulo $2 \pi$ ). Wegen $\bar{\alpha}_{m}=$ $\varphi_{m}+m \pi, \bar{\beta}_{m}=\chi_{m}+m \pi$ und $\varphi_{n+1}-\chi_{n+1}=(-2)^{n+1}(\bar{\alpha}-\bar{\beta})=-2\left(\varphi_{n}-\chi_{n}\right)$ folgt also $\varphi_{m}=\chi_{m}+\pi$ sowie $\varphi_{n}=\chi_{n}$ für $n>m$ (beides modulo $2 \pi$ ). Für alle $n>m$ definieren wir nun $O_{n}$ als $O$ addiert zu den ersten $n$ Gliedern der Reihe des Satzes und $A_{n}, B_{n}, C_{n}$ durch die im Satz angegebenen Gleichungen (für $n \leq m$ sind die entsprechenden Punkte nach dem ersten Teil des Beweises durch die gleichen Formeln gegeben). Mit $O_{n+1}=$ $O_{n}+r \frac{(-1)^{n}}{2^{n+1}}\left(e^{i \varphi_{n}}+e^{i \chi_{n}}+e^{i \psi_{n}}\right)$ und $\varphi_{n+1}=\chi_{n}+\psi_{n}-\varphi_{n}$ ist dann

$$
A_{n+1}=O_{n}+r \frac{(-1)^{n}}{2^{n+1}}\left(e^{i \varphi_{n}}+e^{i \chi_{n}}+e^{i \psi_{n}}-e^{i\left(\chi_{n}+\psi_{n}-\varphi_{n}\right)}\right),
$$

und eine einfache Rechnung ergibt

$$
A_{n+1}-C_{n}=r \frac{(-1)^{n}}{2^{n+1}} e^{-i \varphi_{n}}\left(e^{i \varphi_{n}}-e^{i \psi_{n}}\right)\left(e^{i \varphi_{n}}+e^{i \chi_{n}}\right) .
$$

Mit $\varphi_{m}=\chi_{m}+\pi$ (modulo $2 \pi$ ) folgt hieraus $A_{m+1}=C_{m}$; genauso ist auch $B_{m+1}=C_{m}$. Analog gelten die beiden Gleichungen

$$
\begin{aligned}
& A_{n+1}-B_{n}=r \frac{(-1)^{n}}{2^{n+1}} e^{-i \varphi_{n}}\left(e^{i \varphi_{n}}-e^{i \chi_{n}}\right)\left(e^{i \varphi_{n}}+e^{i \psi_{n}}\right), \\
& B_{n+1}-A_{n}=r \frac{(-1)^{n}}{2^{n+1}} e^{-i \chi_{n}}\left(e^{i \chi_{n}}-e^{i \varphi_{n}}\right)\left(e^{i \chi_{n}}+e^{i \psi_{n}}\right),
\end{aligned}
$$

aus welchen $A_{n+1}=B_{n}, B_{n+1}=A_{n}$ für alle $n>m$ wegen $\varphi_{n}=\chi_{n}$ (modulo $2 \pi$ ) abgelesen werden kann. Folglich ist $A_{n}=B_{n}=C_{m}$ für alle $n>m$. Die Punkte $O_{n}$, die als Grenzpunkt die Reihe des Satzes haben, konvergieren somit nach Definition $\operatorname{der} A_{n}$ auch gegen $C_{m}$ und der Satz ist vollständig bewiesen.

Der Grenzpunkt wird somit sowohl bei unendlichen als auch bei abbrechenden Folgen durch eine gemeinsame Formel beschrieben, in der die beiden Fälle nicht mehr unterschieden werden. Auch wird er dadurch zu einer stetigen Funktion der drei Ecken (bei festem Umkreis).

Satz 2.4. Bezeichnet $M_{c}$ den Mittelpunkt der Seite $c=A B$, so gilt

$$
H_{\infty}=M_{c}+\frac{c}{2} \sum_{n=0}^{\infty}(-1)^{n} \frac{\sin ^{2} 2^{n} \gamma}{2^{n} \sin \gamma} e^{i\left[\omega+(-2)^{n}(\bar{\gamma}-\omega)\right]} .
$$

Falls $H_{n}$ existiert, ergibt der Abbruch der Reihe nach $n$ Gliedern den Mittelpunkt $M_{n}$ der Seite $A_{n} B_{n}$ von $H_{n}$.

Beweis. Falls die gegebene Folge mit $H_{m}$ abbricht, so definieren wir $A_{n}, B_{n}, C_{n}$ und $O_{n}$ für $n>m$ auf die im Satz 2.3 angegebene Weise. Unabhängig davon, ob die Folge abbricht 
oder nicht, ist dann sicherlich $M_{n} \rightarrow H_{\infty}$. Mit dem Satz 2.3 gilt

$$
\begin{aligned}
M_{n+1}-M_{n} & =\frac{A_{n+1}+B_{n+1}}{2}-\frac{A_{n}+B_{n}}{2} \\
& =O_{n+1}+r \frac{(-1)^{n+1}}{2^{n+2}}\left(e^{i \varphi_{n+1}}+e^{i \chi_{n+1}}\right)-O_{n}-r \frac{(-1)^{n}}{2^{n+1}}\left(e^{i \varphi_{n}}+e^{i \chi_{n}}\right) \\
& =r \frac{(-1)^{n+1}}{2^{n+2}}\left(e^{i \varphi_{n+1}}+e^{i \chi_{n+1}}-2 e^{i \psi_{n}}\right) \\
& =r \frac{(-1)^{n+1}}{2^{n+2}}\left(2 \cos \frac{\varphi_{n+1}-\chi_{n+1}}{2} e^{i \frac{\varphi_{n+1}+\chi_{n+1}}{2}}-2 e^{i \psi_{n}}\right) .
\end{aligned}
$$

Zum einen ist $\frac{\varphi_{n+1}+\chi_{n+1}}{2}=\omega-(-2)^{n}(\bar{\alpha}+\bar{\beta}-2 \omega)=\omega-(-2)^{n}(3 \omega-\bar{\gamma}-2 \omega)=\psi_{n}$ und zum anderen $\frac{\varphi_{n+1}-\chi_{n+1}}{2}=-(-2)^{n}(\bar{\alpha}-\bar{\beta})=(-2)^{n+1}( \pm \gamma+k \pi)$, da $\bar{\alpha}-\bar{\beta}=$ $\pm 2 \gamma+2 k \pi$ mit einem bestimmten ganzen $k$ nach dem Peripherie-Zentriwinkelsatz gilt. Mit $1-\cos x=2 \sin ^{2} \frac{x}{2}$ und $r=\frac{c}{2 \sin \gamma}$ (erweiterter Sinussatz) ist also

$$
\begin{aligned}
M_{n+1}-M_{n} & =r \frac{(-1)^{n+1}}{2^{n+2}}\left(2 \cos \left(2^{n+1} \gamma\right) e^{i \psi_{n}}-2 e^{i \psi_{n}}\right) \\
& =\frac{c}{2 \sin \gamma} \cdot(-1)^{n} \frac{1-\cos 2^{n+1} \gamma}{2^{n+1}} e^{i \psi_{n}}=\frac{c}{2} \cdot(-1)^{n} \frac{\sin ^{2} 2^{n} \gamma}{2^{n} \sin \gamma} e^{i \psi_{n}} .
\end{aligned}
$$

Der Satz folgt nun sofort unter Beachtung des Ausdrucks für $\psi_{n}$.

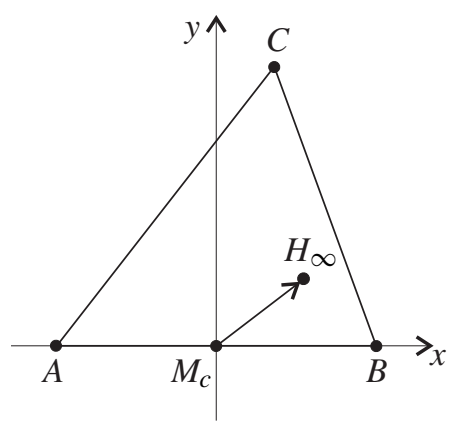

Fig. 3

Legen wir das Ausgangsdreieck speziell so ins Koordinatensystem, dass seine Seite $A B$ parallel zur reellen Achse verläuft (diese Situation ist in Fig. 3 dargestellt; es ist dann $\bar{\alpha}=\frac{3 \pi}{2}-\gamma, \bar{\beta}=-\frac{\pi}{2}+\gamma, \bar{\gamma}=\frac{\pi}{2}+\alpha-\beta$ nach dem Peripherie-Zentriwinkelsatz), so wird die Formel im letzten Satz zu

$$
\overrightarrow{M_{c} H_{\infty}}=i \frac{c}{2} \sum_{n=0}^{\infty}(-1)^{n} \frac{\sin ^{2} 2^{n} \gamma}{2^{n} \sin \gamma} e^{i \frac{1-(-2)^{n+1}}{3}(\alpha-\beta)}
$$

Der Fall $\alpha=\beta$ verdient dabei besonders hervorgehoben zu werden. 
Satz 2.5. Sei $\triangle A B C$ ein gleichschenkliges Dreieck mit der Basis $c$ und dem Winkel $\gamma$ bei $C$. Dann liegt der Grenzpunkt $H_{\infty}$ auf der Symmetrieachse von $\triangle A B C$ und seine Höhe $h_{H_{\infty}}$ über der Basis ist

$$
h_{H_{\infty}}=\frac{c}{2} \sum_{n=0}^{\infty}(-1)^{n} \frac{\sin ^{2} 2^{n} \gamma}{2^{n} \sin \gamma} .
$$

Wie sich herausstellt, ist die durch die Reihe (2.3) dargestellte Funktion von $\gamma$ (natürlich bis auf die Stellen $\gamma=k \pi, k \in \mathbb{Z}$, an welchen sie gar nicht definiert ist) erstaunlicherweise eine überall stetige aber nirgendwo differenzierbare Funktion! Die NichtDifferenzierbarkeit ergibt sich aus dem in [6] bewiesenen Korollar 4.6:

Satz 2.6. Sei $b>1$ und $\mathbb{B}=\left\{b^{n}, n \in \mathbb{Z} \backslash\{0\}\right\}$. Ferner seien $a_{1}, \ldots, a_{m}, m \geq 1$, positive reelle Zahlen mit der Eigenschaft $\frac{a_{k}}{a_{l}} \notin \mathbb{B}$ für alle $k, l=1, \ldots, m$ mit $k \neq l$. Dann ist für jede Funktion $g(x)=c_{1} \cos \left(a_{1} x\right)+\ldots+c_{m} \cos \left(a_{m} x\right)$ mit nicht gleichzeitig verschwindenden Koeffizienten $c_{1}, \ldots, c_{m}$ die zugehörige Funktion

$$
f(x)=\sum_{n=0}^{\infty} \frac{g\left(b^{n} x\right)}{b^{n}}
$$

stetig aber nirgendwo differenzierbar.

Mit $\sin ^{2} x=\frac{1-\cos 2 x}{2}$ lässt sich die den Grenzpunkt beschreibende Funktion als

$$
\begin{aligned}
f(\gamma) & =\frac{c}{2} \sum_{n=0}^{\infty}(-1)^{n} \frac{\sin ^{2} 2^{n} \gamma}{2^{n} \sin \gamma}=\frac{c}{2 \sin \gamma} \sum_{n=0}^{\infty} \frac{(-1)^{n}}{2^{n}}\left(\frac{1}{2}-\frac{\cos 2^{n+1} x}{2}\right) \\
& =\frac{c}{2 \sin \gamma}\left(\frac{1}{3}-\cos \gamma+\sum_{n=0}^{\infty}(-1)^{n} \frac{\cos 2^{n} \gamma}{2^{n}}\right)
\end{aligned}
$$

umschreiben. Die Funktion $\sum_{n=0}^{\infty}(-1)^{n} \frac{\cos 2^{n} \gamma}{2^{n}}$ kann man dabei auch als

$$
\sum_{k=0}^{\infty} \frac{1}{4^{k}}\left(\cos 4^{k} \gamma-\frac{\cos \left(2 \cdot 4^{k} \gamma\right)}{2}\right)=\sum_{k=0}^{\infty} \frac{g\left(4^{k} \gamma\right)}{4^{k}}
$$

mit $g(x):=\cos x-\frac{1}{2} \cos 2 x$ schreiben, so dass diese nach dem oben zitierten Satz 2.6 stetig und nirgendwo differenzierbar ist. Wegen der obigen Beziehung muss das notwendig auch auf $f$ zutreffen (die Stellen $\gamma=k \pi, k \in \mathbb{Z}$, ausgenommen).

\section{Lagebeziehungen des Grenzpunktes}

In diesem Abschnitt werden wir einige Resultate beweisen, die den Höchstabstand des Grenzpunktes zu speziellen Punkten des Ausgangsdreiecks betreffen.

Satz 3.1. Der Abstand des Grenzpunktes $H_{\infty}$ zum Umkreismittelpunkt $O$ ist kleiner oder gleich $\frac{5}{3} r$, wobei $r$ der Umkreisradius ist. 
Beweis. Falls die gegebene Folge endlich ist, definieren wir die Punkte $O_{n}$ wie in Satz 2.3. Wir bestimmen als ersten Schritt das Maximum von $\frac{\overline{O O_{2}}}{r}$. Wir setzen o.B.d.A. $\bar{\gamma}:=$ $-\bar{\alpha}-\bar{\beta}$, also $\varphi_{n}=(-2)^{n} \bar{\alpha}, \chi_{n}=(-2)^{n} \bar{\beta}$ und $\psi_{n}=(-2)^{n} \bar{\gamma}=-\varphi_{n}-\chi_{n}$. Mit den Abkürzungen $y=\frac{\bar{\alpha}+\bar{\beta}}{2}$ und $x=\frac{\bar{\alpha}-\bar{\beta}}{2}$ folgt nach Satz 2.3

$$
\begin{aligned}
\frac{\overrightarrow{O O_{2}}}{r} & =\frac{e^{i \bar{\alpha}}+e^{i \bar{\beta}}+e^{-i(\bar{\alpha}+\bar{\beta})}}{2}-\frac{e^{-2 i \bar{\alpha}}+e^{-2 i \bar{\beta}}+e^{2 i(\bar{\alpha}+\bar{\beta})}}{4} \\
& =\frac{2 \cos x e^{i y}+e^{-2 i y}}{2}-\frac{2 \cos 2 x e^{-2 i y}+e^{4 i y}}{4} \\
& =e^{i y}\left(\cos x+\sin ^{2} x e^{-3 i y}-\frac{1}{4} e^{3 i y}\right) .
\end{aligned}
$$

Betrachtet man $\frac{\overline{O O_{2}}}{r}$ als Funktion von $3 y$ mit dem Parameter $x$, so ergibt eine einfache Rechnung für ihre Extremwerte $\left|\sin ^{2} x-\frac{1}{4} \pm \cos x\right| \leq \frac{5}{4}$, sowie $|\sin x|+\frac{1}{4|\sin x|}$ unter der Bedingung $\sin ^{2} x-\left|\sin ^{2} x-\frac{1}{4}\right||\cos x| \geq 0$. Durch Betrachtung der Ableitungen stellt man unschwer fest, dass $|\sin x|+\frac{1}{4|\sin x|} \leq \frac{5}{4}$ für $\frac{\pi}{10} \leq x \leq \pi-\frac{\pi}{10}$ ist und die Bedingung für den zweiten Extremwert für die übrigen Werte von $x$ aus $[0, \pi]$ nicht erfüllt ist. Also gilt stets $\overline{O_{2}} \leq \frac{5}{4} r$. Hieraus folgt $\overline{O_{n} O_{n+2}} \leq \frac{5}{4} \cdot \frac{r}{2^{n}}$, da der Vektor $\overline{O_{n} O_{n+2}}$ die Form (3.1) mit $\varphi_{n}, \chi_{n}$ anstatt $\bar{\alpha}, \bar{\beta}$ sowie dem Vorfaktor $\frac{(-1)^{n}}{2^{n}}$ hat. Also ist schließlich $\overline{O H_{\infty}} \leq$ $\sum_{n=0,2,4, \ldots} \overline{O_{n} O_{n+2}} \leq \frac{5}{3} r$.

Intensive Computerberechnungen sprechen sehr für die Richtigkeit der folgenden

Vermutung 3.2. Der Abstand von $H_{\infty} z u O$ ist sogar $\leq \frac{4}{3} r$ mit Gleichheit genau für die Anfangswinkel $\frac{\pi}{7}, \frac{2 \pi}{7}, \frac{4 \pi}{7}$.

Einige anfängliche Folgendreiecke für dieses „,heptagonale“ Ausgangsdreieck sind in Fig. 1 rechts dargestellt (siehe [4] für eine umfangreiche Zusammenstellung seiner geometrischen Eigenschaften). Wie am Ende des obigen Beweises würde das aus $\overline{O_{6}} \leq \frac{21}{16} r$ folgen; diese Relation wird ebenso durch massive Computerberechnungen gestützt. Dafür spricht auch, dass die Größe $\frac{1}{r} \overline{O O_{6}}$ für das angegebene Dreieck ein relatives Maximum besitzt (z.B. als Funktion von $\bar{\alpha}$ und $\bar{\beta}$ mit $\bar{\gamma}:=-\bar{\alpha}-\bar{\beta}$ für $\bar{\alpha}=\frac{2 \pi}{7}, \bar{\beta}=\frac{4 \pi}{7}$ ); dabei ist anzumerken, dass das zweite Folgendreieck $H_{2}$ aus $H_{0}$ durch Streckung mit Faktor $1 / 4$ hervorgeht und sein Umkreismittelpunkt $\mathrm{O}_{2}$ auf dem Umkreis von $H_{0}$ liegt, so dass die Vektoren $\overrightarrow{\mathrm{O}_{0} \mathrm{O}_{2}}, \overrightarrow{\mathrm{O}_{2} \mathrm{O}_{4}}, \ldots$ alle gleichgerichtet sind und Längen $r, \frac{r}{4}, \frac{r}{4^{2}}, \ldots$ haben. Für spitze Ausgangsdreiecke folgt die Vermutung $\overline{O H_{\infty}} \leq \overline{O O_{1}}+\overline{O_{1} H_{\infty}} \leq \frac{1}{2} r+\frac{5}{3} \cdot \frac{r}{2}=\frac{4}{3} r$ mit dem soeben bewiesenen Satz, da der Höhenschnittpunkt $P$ innerhalb des Umkreises liegt und $O_{1}$ der Mittelpunkt zwischen $O$ und $P$ ist. Der beste Vorfaktor nur für spitze Ausgangsdreiecke ist vermutlich 1,04752. ., angenommen für die Anfangswinkel $\frac{3}{56} \pi, \frac{26}{56} \pi, \frac{27}{56} \pi$; interessanterweise ist hier $\mathrm{H}_{3}$ das heptagonale Dreieck von oben.

Satz 3.3. Der Abstand von $H_{\infty}$ zu der Mitte einer Seite des Ausgangsdreiecks ist kleiner als $\frac{1}{2} \sum_{n=-\infty}^{\infty} \frac{\sin ^{2}\left(2^{n} \pi / 3\right)}{2^{n} \pi / 3}=1,213 \ldots$ multipliziert mit der Länge dieser Seite. 
Beweis. Unter Benutzung von Satz 2.4 ist

$$
\overline{M_{c} H_{\infty}} \leq \frac{c}{2} \sum_{n=0}^{\infty} \frac{\sin ^{2} 2^{n} \gamma}{2^{n} \sin \gamma}=: \frac{c}{2} f(\gamma)
$$

Für $m \geq 0$ ist nach einer einfachen Umformung zunächst

$$
f\left(\frac{\gamma}{2^{m}}\right)=\frac{\gamma}{2^{m} \sin \frac{\gamma}{2^{m}}}\left(\sum_{n=1}^{m} \frac{2^{n}}{\gamma} \sin ^{2} \frac{\gamma}{2^{n}}+\sum_{n=0}^{\infty} \frac{\sin ^{2} 2^{n} \gamma}{2^{n} \gamma}\right)
$$

Für alle $0<\gamma \leq \frac{\pi}{2}$ ist weiter

$$
f(\gamma) \leq \sum_{n=0}^{\infty} \frac{1}{2^{n} \sin \gamma}=\frac{2}{\sin \gamma}=\frac{\sin \frac{\gamma}{2}}{\left(1-\cos \frac{\gamma}{2}\right)\left(1+\cos \frac{\gamma}{2}\right) \cos \frac{\gamma}{2}}<\frac{\sin \frac{\gamma}{2}}{1-\cos \frac{\gamma}{2}}
$$

da $\left(1+\cos \frac{\gamma}{2}\right) \cos \frac{\gamma}{2}$ auf $\left[0, \frac{\pi}{2}\right]$ monoton fällt und bei $\gamma=\frac{\pi}{2}$ den Wert $\frac{1}{2}+\frac{1}{\sqrt{2}}>1$ hat. Die Beziehung

$$
f\left(\frac{\gamma}{2}\right)=\sin \frac{\gamma}{2}+\sum_{n=1}^{\infty} \frac{\sin ^{2} 2^{n} \frac{\gamma}{2}}{2^{n} \sin \frac{\gamma}{2}}=\sin \frac{\gamma}{2}+\frac{\sin \gamma}{2 \sin \frac{\gamma}{2}} f(\gamma)=\sin \frac{\gamma}{2}+\cos \frac{\gamma}{2} f(\gamma)
$$

führt damit zu $f\left(\frac{\gamma}{2}\right)-f(\gamma)>0$ bzw. $f(\gamma)<f\left(\frac{\gamma}{2}\right)$, und iterativ auch $f\left(\frac{\gamma}{2^{m}}\right)<f\left(\frac{\gamma}{2^{k}}\right)$ für $0 \leq m<k$. Mit (3.2) bedeutet das

$$
f\left(\frac{\gamma}{2^{m}}\right)<\lim _{k \rightarrow \infty} f\left(\frac{\gamma}{2^{k}}\right)=\sum_{n=1}^{\infty} \frac{2^{n}}{\gamma} \sin ^{2} \frac{\gamma}{2^{n}}+\sum_{n=0}^{\infty} \frac{\sin ^{2} 2^{n} \gamma}{2^{n} \gamma}=g(\gamma)+\frac{h(\gamma)}{\gamma}
$$

für alle $m \geq 0,0<\gamma \leq \frac{\pi}{2}$ mit $g(x):=\sum_{n=1}^{\infty} \frac{2^{n}}{x} \sin ^{2} \frac{x}{2^{n}}$ und $h(x):=\sum_{n=0}^{\infty} \frac{\sin ^{2} 2^{n} x}{2^{n}}$.

Im Folgenden werden wir $g(\gamma)+\frac{h(\gamma)}{\gamma} \leq g\left(\frac{\pi}{3}\right)+\frac{h(\pi / 3)}{\pi / 3}=\sum_{n=-\infty}^{\infty} \frac{\sin ^{2}\left(2^{n} \pi / 3\right)}{2^{n} \pi / 3}=: \kappa$ für $\frac{\pi}{4} \leq \gamma \leq \frac{\pi}{2}$ zeigen. Variiert dann $\gamma$ im Intervall $\left[\frac{\pi}{4}, \frac{\pi}{2}\right]$, so variiert $\frac{\gamma}{2^{m}}$ für $m=0,1,2, \ldots$ im Intervall $\left[\frac{\pi}{4}, \frac{\pi}{2}\right],\left[\frac{\pi}{8}, \frac{\pi}{4}\right],\left[\frac{\pi}{16}, \frac{\pi}{8}\right]$ usw., d.h. insgesamt wird so $f(\gamma)<\kappa$ für $0<\gamma \leq \frac{\pi}{2}$ nachgewiesen werden. Mit der leicht nachprüfbaren Relation $f(\pi-\gamma)=f(\gamma)$ ergibt dies gerade die Behauptung des Satzes.

Für alle $x$ gilt offenbar die Beziehung $h(x)=\sin ^{2} x+\frac{1}{2} h(2 x)$ (speziell folgt $h\left(\frac{\pi}{3}\right)=\frac{3}{2}$ wegen $h(x)=h(\pi-x)$ ) und mit dieser auch $h(x)=\sin ^{2} x+\frac{1}{2} \sin ^{2} 2 x+\frac{1}{4} h(4 x)$. Nimmt also die Funktion $h$ an einer Stelle $x_{0}$ ihr Maximum $M$ an, so folgt $M=h\left(x_{0}\right) \leq$ $\sin ^{2} x_{0}+\frac{1}{2} \sin ^{2} 2 x_{0}+\frac{1}{4} M$, d.h.

$$
M \leq \frac{4}{3} \sin ^{2} x_{0}+\frac{2}{3} \sin ^{2} 2 x_{0}=\frac{4}{3} \sin ^{2} x_{0}+\frac{8}{3} \sin ^{2} x_{0} \cos ^{2} x_{0}=\frac{3}{2}-\frac{8}{3}\left(\sin ^{2} x_{0}-\frac{3}{4}\right)^{2} \text {. }
$$

Also ist $h(x) \leq \frac{3}{2}=h\left(\frac{\pi}{3}\right)$ für alle $x$ und zudem $h(x)=\sin ^{2} x+\frac{1}{2} h(2 x) \leq \sin ^{2} x+\frac{3}{4}$. 
Die Funktion $d_{1}(x):=\sin ^{2} x+\frac{3}{4}-x\left(\frac{13}{2 \pi}-\frac{6}{\pi^{2}} x\right)$ erfüllt $d_{1}\left(\frac{\pi}{4}\right)=d_{1}\left(\frac{\pi}{3}\right)=0$ und ist auf $I_{1}:=\left[\frac{\pi}{4}, \frac{\pi}{3}\right]$ wegen $d_{1}^{\prime \prime}(x)=2 \cos 2 x+\frac{12}{\pi^{2}}>0$ konvex. Folglich ist $d_{1}(x) \leq 0$ auf $I_{1}$, d.h. $\frac{1}{x}\left(\sin ^{2} x+\frac{3}{4}\right) \leq \frac{13}{2 \pi}-\frac{6}{\pi^{2}} x$. Weiterhin ist $d_{2}(x):=\frac{3}{2 x}$ auf $I_{2}:=\left[\frac{\pi}{3}, \frac{\pi}{2}\right]$ wegen $d_{2}^{\prime \prime}(x)=\frac{3}{x^{3}}>0$ konvex, kann also nach oben durch die lineare Funktion, welche mit $d_{2}$ in den Endpunkten übereinstimmt, d.h. $\frac{15}{2 \pi}-\frac{9}{\pi^{2}} x$ abgeschätzt werden.

Wir haben somit $g(x)+\frac{h(x)}{x} \leq g(x)+\frac{13}{2 \pi}-\frac{6}{\pi^{2}} x$ auf $I_{1}$ und $g(x)+\frac{h(x)}{x} \leq g(x)+\frac{15}{2 \pi}-\frac{9}{\pi^{2}} x$ auf $I_{2}$, wobei für $x=\frac{\pi}{3}$ offenbar jeweils Gleichheit eintritt. Es gilt

$$
\begin{aligned}
& \qquad g^{\prime}(x)=\sum_{n=1}^{\infty}\left(\frac{1}{x} \sin \frac{x}{2^{n-1}}-\frac{2^{n}}{x^{2}} \sin ^{2} \frac{x}{2^{n}}\right)=\frac{\sin x}{x}+\frac{1}{x} \sum_{n=1}^{\infty}\left(\sin \frac{x}{2^{n}}-\frac{\sin ^{2} \frac{x}{2^{n}}}{\frac{x}{2^{n}}}\right), \\
& \text { wegen } 0<\sin t-\frac{\sin ^{2} t}{t}=\sin t\left(1-\frac{\sin t}{t}\right)<t\left(1-\frac{t-t^{3} / 6}{t}\right)=\frac{t^{3}}{6} \text { für } 0<t<\pi \text { also } \\
& \qquad g^{\prime}(x)>\frac{\sin x}{x} \geq \frac{\sin \frac{\pi}{3}}{\frac{\pi}{3}}=\frac{3 \sqrt{3}}{2 \pi}>\frac{6}{\pi^{2}} \quad \text { auf } I_{1}, \\
& g^{\prime}(x)<\frac{\sin x}{x}+\frac{1}{x} \sum_{n=1}^{\infty} \frac{\left(\frac{x}{2^{n}}\right)^{3}}{6}=\frac{\sin x}{x}+\frac{x^{2}}{42} \leq \frac{\sin \frac{\pi}{3}}{\frac{\pi}{3}}+\frac{\left(\frac{\pi}{2}\right)^{2}}{42}<\frac{9}{\pi^{2}} \text { auf } I_{2} .
\end{aligned}
$$

Damit ist $g(x)+\frac{13}{2 \pi}-\frac{6}{\pi^{2}} x$ monoton wachsend auf $I_{1}$ und $g(x)+\frac{15}{2 \pi}-\frac{9}{\pi^{2}} x$ monoton fallend auf $I_{2}$, d.h. die beiden Funktionen werden jeweils für $\frac{\pi}{3}$ mit dem gemeinsamen Wert $g\left(\frac{\pi}{3}\right)+\frac{h(\pi / 3)}{\pi / 3}$ maximal, womit insgesamt $g(x)+\frac{h(x)}{x} \leq g\left(\frac{\pi}{3}\right)+\frac{h(\pi / 3)}{\pi / 3}$ auf $I_{1} \cup I_{2}=$ $\left[\frac{\pi}{4}, \frac{\pi}{2}\right]$ gezeigt ist.

Der kleinste Vorfaktor für die Aussage des Satzes liegt vermutlich nah bei 0,785786 (dies ist der Wert für $\gamma=\frac{2,69446565}{2^{10}}, \alpha-\beta=0,6328764878$ ). Diesen Wert zu finden ist deutlich schwieriger als beim Abstand zum Umkreismittelpunkt: Während man für jenes Problem ein Anfangsdreieck angeben konnte, das (höchstwahrscheinlich) zur optimalen Konstanten führt, scheinen hier die größeren Werte von $\overline{M_{c} H_{\infty}} / c$ Computerexperimenten zufolge erst bei sehr kleinen Werten von $\gamma$ vorzukommen. Die Funktion im Satz 2.4 verhält sich jedoch gerade in diesem Bereich - obwohl mit scheinbar periodischen Mustern - höchstgradig chaotisch.

Satz 3.4. Der Abstand von $H_{\infty}$ zu der Ecke A ist kleiner als $\lambda r|\cos \alpha|$ mit $\lambda=1+$ $\sum_{n=-\infty}^{\infty} \frac{\sin ^{2}\left(2^{n} \pi / 3\right)}{2^{n} \pi / 3}=3,426 \ldots$ (und entsprechend für die anderen Ecken).

Beweis. Für spitzes $\triangle A B C$ (der andere Fall lässt sich analog behandeln) entnimmt man der Skizze $\overline{A C_{H}}=b \cos \alpha=\overline{A H} \cos \left(\frac{\pi}{2}-\beta\right)=\overline{A H} \sin \beta$, d.h. mit dem Sinussatz $\overline{A H}=b \frac{\cos \alpha}{\sin \beta}=2 r \cos \alpha$. Da das Dreieck $\triangle A B H$ offensichtlich das gleiche Höhenfußpunktdreieck wie $\triangle A B C$ hat, ist der Grenzpunkt $H_{\infty}$ für beide Ausgangsdreiecke der gleiche. Für den Abstand von $H_{\infty}$ zu der Mitte $M$ der Seite $A H$ gilt $\overline{M H_{\infty}}<\frac{\lambda-1}{2} \overline{A H}$ nach Satz 3.3. Mit $\overline{A H}=2 r \cos \alpha$ folgt somit

$$
\overline{A H_{\infty}} \leq \overline{A M}+\overline{M H_{\infty}}=\frac{1}{2} \overline{A H}+\overline{M H_{\infty}}<\frac{1}{2} \overline{A H}+\frac{\lambda-1}{2} \overline{A H}=\lambda r \cos \alpha .
$$




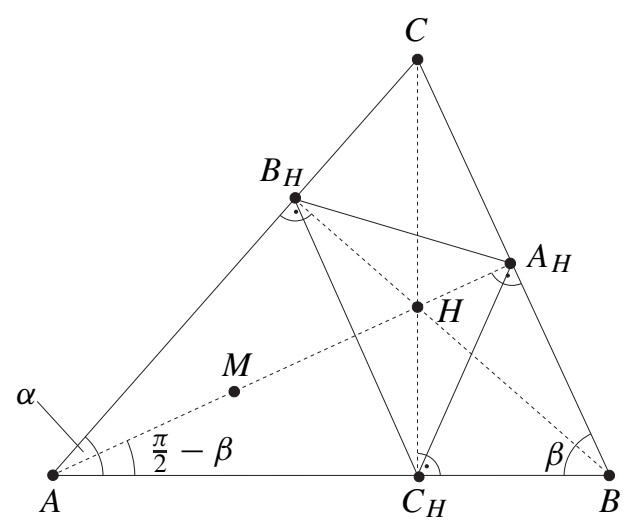

Fig. 4 Skizze zum Beweis von Satz 3.4

Besitzt das Ausgangsdreieck also einen fast rechten Winkel, so liegt der Grenzpunkt stets sehr nahe an der entsprechenden Ecke, was nochmals für die Natürlichkeit unserer Definition des Grenzpunktes für abbrechende Folgen spricht. Der Herleitung zufolge ist der kleinste Vorfaktor für diesen Satz vermutlich genau $1+2 \kappa$, wenn $\kappa$ der kleinste Vorfaktor für den Satz 3.3 ist.

\section{Die Winkel der Folgendreiecke}

\subsection{Die Verhältnisgrößen}

Mit dem letzten Abschnitt beenden wir die Untersuchung des Grenzpunktes und wenden uns nunmehr den Folgendreiecken an sich, und zwar ihren Innenwinkeln $\alpha_{n}, \beta_{n}, \gamma_{n}$ zu. Als Erstes werden wir das wichtige Fortsetzbarkeitskriterium und die expliziten Formeln für die $n$-ten Winkel herleiten, daneben stellen wir auch einige weitere interessante Resultate rund um die Winkel der Folgendreiecke zusammen.

Die Innenwinkel $\alpha, \beta, \gamma$ unseres Ausgangsdreiecks $H_{0}=\triangle A B C$ schreiben wir in der Form $\alpha=a \pi, \beta=b \pi$ und $\gamma=c \pi$. Die Verhältnisgrößen $a, b, c$ liegen dann zwischen 0 und 1 und erfüllen $a+b+c=1$. Die Binärdarstellung dieser Zahlen wird für uns im Folgenden zu einem äußerst mächtigen Hilfsmittel werden:

$$
a=0, a_{0} a_{1} a_{2} a_{3} \ldots, \quad b=0, b_{0} b_{1} b_{2} b_{3} \ldots, \quad c=0, c_{0} c_{1} c_{2} c_{3} \ldots
$$

Die Zählung der Nachkommaziffern fangen wir dabei bei 0 an. Wenn wir im Folgenden eine endliche oder unendliche Entwicklung aufschreiben, so meinen wir damit immer die Binärdarstellung der entsprechenden Zahl. Die Periode $\overline{1}$ wird ausgeschlossen. Die Verhältnisgrößen von $H_{n}$ für $n=0,1,2, \ldots$ werden wir mit $a^{(n)}, b^{(n)}, c^{(n)}$ bezeichnen.

Wir bemerken zunächst das folgende einfache aber grundlegende Ergebnis aus der Elementargeometrie: Ist $H_{n}$ spitz, so ist $\alpha_{n+1}=\pi-2 \alpha_{n}, \beta_{n+1}=\pi-2 \beta_{n}, \gamma_{n+1}=\pi-2 \gamma_{n}$. Ist $H_{n}$ hingegen stumpf, so ist $\alpha_{n+1}=2 \alpha_{n}, \beta_{n+1}=2 \beta_{n}, \gamma_{n+1}=2 \gamma_{n}-\pi$, wenn der stumpfe Winkel bei $C_{n}$ liegt (und entsprechend für die anderen Ecken). 
In die Verhältnisgrößen ,übersetzt" bedeutet das: $a^{(n+1)}, b^{(n+1)}, c^{(n+1)}$ entstehen aus $a^{(n)}$, $b^{(n)}, c^{(n)}$ durch das Vorrücken des Kommas um eine Stelle nach rechts, sowie durch eine anschließende Ziffernumkehrung im Falle $a_{0}^{(n)}=b_{0}^{(n)}=c_{0}^{(n)}$ (Nullen werden zu Einsen gemacht und umgekehrt). Iterativ ergibt sich hieraus auch die folgende allgemeine Regel, mit der sich die Verhältnisgrößen von $H_{n}$ und damit auch die entsprechenden Innenwinkel direkt berechnen lassen.

Satz 4.1. Sind $H_{0}, \ldots, H_{n-1}$ nichtrechtwinklig, so ergeben sich die Binärentwicklungen von $a^{(n)}, b^{(n)}, c^{(n)}$ aus denen von $a, b$, c jeweils durch Vorrücken des Kommas um $n$ Stellen nach rechts, wobei anschließend die Ziffern umgekehrt werden, falls die Anzahl der Stellen $0 \leq i \leq n-1$ mit $a_{i}=b_{i}=c_{i}$ ungerade ist.

Beispiel. Gegeben seien z.B. $\alpha=\frac{15}{34} \pi, \beta=\frac{11}{34} \pi, \gamma=\frac{4}{17} \pi$ und gesucht die Innenwinkel von $\mathrm{H}_{5}$. Es ist

$$
\begin{array}{lll}
a=\frac{15}{34}=0,01110 \overline{00011110} \ldots & a^{(5)}=0, \overline{00011110} \ldots=\frac{2}{17} \\
b=\frac{11}{34}=0,01010 \overline{01011010} \ldots & \text { und damit } & b^{(5)}=0, \overline{01011010} \ldots=\frac{6}{17} \\
c=\frac{4}{17}=0,00111 \overline{10000111} \ldots & c^{(5)}=0, \overline{10000111} \ldots=\frac{9}{17},
\end{array}
$$

denn unter den ersten 5 Stellen $i$ gibt es genau 2 mit jeweils drei gleichen Ziffern; da 2 gerade ist, rücken wir das Komma um 5 Stellen nach rechts ohne anschließend die Ziffern umzukehren. Also ist $\alpha_{5}=\frac{2}{17} \pi, \beta_{5}=\frac{6}{17} \pi$ und $\gamma_{5}=\frac{9}{17} \pi$.

Satz 4.2 (Fortsetzbarkeitskriterium). Eine Folge von Höhenfußpunktdreiecken ist genau dann unendlich, wenn keine der drei Binärentwicklungen von $a, b$, $c$ abbricht.

Beweis. Ist keine der Entwicklungen von $a, b, c$ abbrechend, so ist insbesondere keine dieser Größen gleich 0,1 und $H_{0}$ nichtrechtwinklig. Mit Satz 4.1 brechen auch die Entwicklungen von $a^{(1)}, b^{(1)}, c^{(1)}$ nicht ab und $H_{1}$ ist nichtrechtwinklig usw. Damit ist kein Folgendreieck rechtwinklig.

Sind umgekehrt z.B. in der Binärentwicklung von $a$ höchstens die ersten $n$ Ziffern von 0 verschieden, würde Satz 4.1 den Widerspruch $a^{(n)}=0$ oder $a^{(n)}=1$ ergeben, wenn die Folge keine rechtwinkligen Dreiecke enthalten würde.

Satz 4.1 erlaubt uns auf direkte Weise die allgemeine Gestalt der Verhältnisgrößen $a, b, c$ aufzudecken:

Satz 4.3. Bei unendlichen Folgen stehen an den Stellen mit drei gleichen Ziffern mit 0 beginnend abwechselnd 0 und 1, die übrigen Stellen enthalten genau eine 1, wenn sie direkt nach dem Komma oder einer Stelle mit drei Einsen folgen, bzw. genau eine 0, wenn sie nach einer Stelle mit drei Nullen folgen, also etwa

$$
\begin{aligned}
& a=0,100 \ldots 100 \quad 0 \quad 101 \ldots 0101100 \ldots 100 \quad 0 \quad 101 \ldots 0101 \ldots \text {, } \\
& b=0,010 \ldots 010 \quad 0 \quad 111 \ldots 101 \quad 1 \quad 010 \ldots 01000111 \ldots 101 \quad 1 \ldots \text {, } \\
& c=0,001 \ldots 001 \quad 0 \quad 010 \ldots 111 \quad 1 \quad 001 \ldots 0010010 \ldots 111 \quad 1 \ldots
\end{aligned}
$$


Verhältnisgrößen endlicher Folgen entsprechen der obigen Beschreibung, wenn man sie mit Periode $\overline{1}$ aufschreibt.

Beweis. Im Fall einer unendlichen Folge $H_{0}, H_{1}, H_{2}, \ldots$ sei nämlich $n \geq 0$ und $g_{n}$ die Anzahl der Stellen $0 \leq i \leq n-1$ mit $a_{i}=b_{i}=c_{i}$. Nach Satz 4.1 ist dann $\left(a_{0}^{(n)}, b_{0}^{(n)}, c_{0}^{(n)}\right)=\left(a_{n}, b_{n}, c_{n}\right)$ für gerades $g_{n}$ und $\left(a_{0}^{(n)}, b_{0}^{(n)}, c_{0}^{(n)}\right)=\left(1-a_{n}, 1-\right.$ $\left.b_{n}, 1-c_{n}\right)$ für ungerades $g_{n}$. Bei spitzem $H_{n}$ ist $a_{0}^{(n)}=b_{0}^{(n)}=c_{0}^{(n)}=0$ und somit $a_{n}=b_{n}=c_{n}=0$ für $g_{n}$ gerade und $a_{n}=b_{n}=c_{n}=1$ für $g_{n}$ ungerade. Bei stumpfem $H_{n}$ ist z.B. $\left(a_{0}^{(n)}, b_{0}^{(n)}, c_{0}^{(n)}\right)=(1,0,0)$, wenn der stumpfe Winkel bei $A_{n}$ liegt, also $\left(a_{n}, b_{n}, c_{n}\right)=(1,0,0)$ für $g_{n}$ gerade und $\left(a_{n}, b_{n}, c_{n}\right)=(0,1,1)$ für $g_{n}$ ungerade. Die obige Beschreibung (4.1) ergibt sich, wenn wir jetzt nacheinander die Stellen $n$ mit $g_{n}=0,1,2, \ldots$ betrachten. Bei Folgen, die mit einem rechtwinkligen Dreieck $H_{n_{0}}$ abbrechen, ist die Begründung für die Stellen $n<n_{0}$ genau wie oben und für $n \geq n_{0}$ ganz analog.

Drei beliebige nach dieser Vorschrift konstruierte Zahlen $a, b, c$, erfüllen umgekehrt, wie man leicht feststellt, automatisch die Bedingung $a+b+c=1$, so dass sie im Fall $0<a, b, c<1$ Verhältnisgrößen eines Dreiecks darstellen. Damit haben wir eine ganz bequeme Methode, um alle möglichen Belegungen für die jeweils ersten $n$ Stellen zu ermitteln, was z.B. im Abschnitt 4.3 von Nutzen sein wird.

Betrachten wir die jeweils aus den ersten $n$ Ziffern entstehenden ganzen Zahlen $u=$ $a_{0} \ldots a_{n-1}, v=b_{0} \ldots b_{n-1}$ und $w=c_{0} \ldots c_{n-1}$, so offenbart uns die Darstellung (4.1) im Falle der Existenz von $H_{n}$ sogleich, dass die Summe $s=u+v+w$ entweder gleich $11 \ldots 11=2^{n}-1$ oder $11 \ldots 10=2^{n}-2$ (jeweils $n$ Ziffern) ist, je nach dem, ob $g_{n}$ gerade oder ungerade ist. Die Entscheidungsgröße $g_{n}$ im Satz 4.1 ist also gerade oder ungerade, je nach dem, ob $s$ ungerade oder gerade ist und wir gewinnen zusammen mit Kriterium 4.2 den folgenden bemerkenswerten Satz, der uns die $n$-ten Verhältnisgrößen direkt und ohne die Kenntnis der jeweiligen Binärdarstellungen oder sonstige Fallunterscheidungen liefert $(\lfloor x\rfloor$ ist der ganzzahlige und $\{x\}=x-\lfloor x\rfloor$ der gebrochene Anteil von $x)$ :

Satz 4.4. Für $n>0$ sind die Verhältnisgrößen von $H_{n}$ gegeben durch

$$
\frac{1}{2}+(-1)^{s}\left(\frac{1}{2}-\left\{2^{n} a\right\}\right), \quad \frac{1}{2}+(-1)^{s}\left(\frac{1}{2}-\left\{2^{n} b\right\}\right), \quad \frac{1}{2}+(-1)^{s}\left(\frac{1}{2}-\left\{2^{n} c\right\}\right)
$$

mit $s=\left\lfloor 2^{n} a\right\rfloor+\left\lfloor 2^{n} b\right\rfloor+\left\lfloor 2^{n} c\right\rfloor$, falls $\left\{2^{n} a\right\},\left\{2^{n} b\right\},\left\{2^{n} c\right\}>0$. Ansonsten existiert $H_{n}$ nicht.

(Die bei Hobson [7] sowie in der Erläuterung von Hatzipolakis zur Folge A001045 in [10] angegebenen Formeln $\alpha_{n}=\frac{1-(-2)^{n}}{3} \pi+(-2)^{n} \alpha$ usw. sind nicht richtig, worauf im ersten Fall schon Kingston und Synge [8] hingewiesen haben.)

\subsection{Spitze und stumpfe Folgendreiecke}

In der Tat haben wir im letzten Abschnitt sogar mehr bewiesen als eine Beschreibung, wir haben auch die Bedeutung der Ziffern erfasst: 
Satz 4.5. Wenn $H_{n}$ existiert und nichtrechtwinklig ist, ist $H_{n}$ spitz genau dann, wenn $a_{n}=$ $b_{n}=c_{n}$, und stumpf bei $A_{n}$ genau dann, wenn $\left(a_{n}, b_{n}, c_{n}\right)=(1,0,0)$ oder $\left(a_{n}, b_{n}, c_{n}\right)=$ $(0,1,1)$ (und entsprechend für $B_{n}$ und $C_{n}$ ).

Damit können wir nicht nur zu gegebenen $a, b, c$ sofort das Spitz-Stumpf-Verhalten ablesen, sondern wir können verblüffenderweise auch umgekehrt zu fast jeder Vorgabe der Spitz-Stumpf-Eigenschaft (für jedes $n$ wird vorgegeben, ob $H_{n}$ spitz oder stumpf bei $A_{n}$, $B_{n}$ oder $C_{n}$ sein soll; oder aber nur für $n<n_{0}$ und dass $H_{n_{0}}$ rechtwinklig sein soll) ein Ausgangsdreieck finden, welches diese Vorgabe erfüllt. Wir müssen die Ziffern von $a, b, c$ bloß so entsprechend dem obigen Satz wählen, dass die allgemeine Struktur (4.1) gewährleistet ist. Bei abbrechenden Folgen sind alle Vorgaben realisierbar, sogar so, dass alle drei Verhältnisgrößen die Form $\frac{k}{2^{n}} \pi$ haben. Bei unendlichen sind nur diejenigen ausgenommen, die zu Perioden $\overline{0}$ oder $\overline{1}$ führen, d.h. Vorgaben mit nur endlich vielen spitzen Dreiecken und bei denen zusätzlich der stumpfe Winkel ab einem gewissen $n_{0}$ nicht mehr bei $A_{n}, B_{n}$ bzw. $C_{n}$ liegen soll. Mehr noch: Im Fall einer unendlichen Folge ist das Ausgangsdreieck durch eine realisierbare Vorgabe der Spitz-Stumpf-Eigenschaft aller Folgendreiecke offenbar eindeutig bestimmt! Diese Korrelation ist das Hauptresultat von Alexander [1] und führt zu der eingangs erwähnten Kodierung. Wenn man aber nur vorschreibt, ob $H_{n}$ spitz oder stumpf sein soll, ist jede solche Vorgabe realisierbar, bei unendlich vielen stumpfen Dreiecken sogar auf überabzählbar viele Weisen!

Wir bemerken speziell, dass die ersten $n$ Folgendreiecke $H_{0}, H_{1}, \ldots, H_{n-1}$ genau dann alle spitz sind, wenn die Entwicklungen von $a, b, c$ wie folgt anfangen:

$$
\begin{aligned}
& a=0, \underbrace{01010 \ldots}_{n \text { Ziffern }} a_{n} a_{n+1} \ldots, \\
& b=0, \underbrace{01010 \ldots}_{n \text { Ziffern }} b_{n} b_{n+1} \ldots, \\
& c=0, \underbrace{01010 \ldots}_{n \text { Ziffern }} c_{n} c_{n+1} \ldots,
\end{aligned}
$$

Äquivalent kann man auch $-\frac{\pi}{3 \cdot 2^{n-u}}<\alpha-\frac{\pi}{3}, \beta-\frac{\pi}{3}, \gamma-\frac{\pi}{3}<\frac{\pi}{3 \cdot 2^{n-v}}$ mit $(u, v)=$ $(0,1)$ für gerades $n$ und $(u, v)=(1,0)$ für ungerades $n$ fordern, wie man leicht mit Hilfe der am Anfang bemerkten Rekursionsformeln für die Innenwinkel zeigt. Sind also alle Folgendreiecke spitz, so ist das Ausgangsdreieck $H_{0}$ gleichseitig, d.h. fängt eine Folge von Höhenfußpunktdreiecken mit einem nichtgleichseitigen Dreieck an, so kommt in ihr auf jeden Fall ein stumpfes Dreieck vor.

\subsection{Periodische Folgen}

Wir nennen eine Folge von Höhenfußpunktdreiecken $n$-periodisch ( $n \geq 1)$, falls das $n$ te Folgendreieck $H_{n}$ zum Anfangsdreieck $H_{0}$ ähnlich ist. Im speziellen Falle $\alpha_{n}=\alpha$, $\beta_{n}=\beta, \gamma_{n}=\gamma$ nennen wir die Folge streng $n$-periodisch. Bei periodischen Folgen wiederholen sich also immer die gleichen Dreiecke bis auf Ähnlichkeit: $H_{n}$ ist zu $H_{0}$ ähnlich, $H_{n+1}$ zu $H_{1}, H_{n+2}$ zu $H_{2}$ usw. 
Die Ziffern der untereinander aufgeschriebenen Binärentwicklungen der Verhältnisgrößen $a, b, c$ fassen wir zu Blöcken von jeweils $n$ Spalten zusammen:

$$
\begin{aligned}
& a=0, \quad a_{0} a_{1} a_{2} \ldots a_{n-1} \quad a_{n} a_{n+1} \ldots a_{2 n-1} \quad a_{2 n} \ldots, \\
& b=0, \quad b_{0} b_{1} b_{2} \ldots b_{n-1} \quad b_{n} b_{n+1} \ldots b_{2 n-1} \quad b_{2 n} \ldots \text {, } \\
& c=0, \quad \underbrace{c_{0} c_{1} c_{2} \ldots c_{n-1}}_{\text {erster } n \text {-Block }} \quad \underbrace{c_{n} c_{n+1} \ldots c_{2 n-1}}_{\text {zweiter } n \text {-Block }} c_{2 n} \ldots
\end{aligned}
$$

Der folgende Satz, welcher sich auf eine einfache Weise mit dem Satz 4.1 beweisen lässt, gibt ein notwendiges und hinreichendes Kriterium für die $n$-Periodizität.

Satz 4.6. Eine Dreiecksfolge ist genau dann n-periodisch, $n \geq 1$, wenn jeder $n$-Block aus dem vorhergehenden durch irgendeine, für jeden $n$-Block die gleiche Vertauschung der drei Zeilen, sowie durch die Ziffernumkehrung, falls die Anzahl der Stellen $0 \leq i \leq n-1$ mit $a_{i}=b_{i}=c_{i}$ ungerade ist, hervorgeht. Für strenge Periodizität ist die Zeilenvertauschung wegzulassen.

Beispiel. Für die Anfangswinkel $\alpha=\frac{\pi}{7}, \beta=\frac{2 \pi}{7}, \gamma=\frac{4 \pi}{7}$ ist

$$
\begin{aligned}
& a=\frac{1}{7}=0,001001001 \ldots, \\
& b=\frac{2}{7}=0,010010010 \ldots, \\
& c=\frac{4}{7}=0,100100100 \ldots
\end{aligned}
$$

Die entsprechende Folge ist also 1-periodisch und streng 3-periodisch (dieser Folge begegneten wir schon im Abschnitt 3).

Um eine $n$-periodische Folge zu erhalten, müssen wir also bloß beliebig die Ziffern an den jeweils ersten $n$ Stellen gemäß dem Aufbau (4.1) vorgeben sowie irgendeine Zeilenpermutation wählen, mit der dann auch die übrigen Ziffern entsprechend dem obigen Satz bestimmt werden. Die eventuell periodischen Folgen, d.h. solche, bei denen erst nach einer anfänglichen Verzögerung von $d$ Schritten die mit $H_{d}$ beginnende Folge periodisch ist, können offensichtlich ganz analog beschrieben und auch konstruiert werden, indem der erste $n$-Block erst ab Spalte $d$ begonnen wird.

Wir bemerken, dass nach obigem Satz jede $n$-periodische Folge auch streng $6 n$-periodisch ist. Da sich in den Entwicklungen von $a, b, c$ immer die gleichen Ziffern von $n$-Block zu $n$-Block ggf. bis auf Ziffernumkehrung wiederholen, kann man auch die entsprechenden Darstellungen als ,gewöhnliche“ Brüche $\frac{x}{y}$ angeben. Wegen

$$
\begin{aligned}
& \left(0, a_{0} a_{1} a_{2} \ldots a_{n-1} a_{0} a_{1} a_{2} \ldots a_{n-1} a_{0} \ldots\right)=\frac{p}{2^{n}-1} \quad \text { und } \\
& \left(0, a_{0} a_{1} a_{2} \ldots a_{n-1} \bar{a}_{0} \bar{a}_{1} \bar{a}_{2} \ldots \bar{a}_{n-1} a_{0} \ldots\right)=\frac{p+1}{2^{n}+1}
\end{aligned}
$$

mit $\bar{a}_{i}:=1-a_{i}$ und $p:=a_{0} a_{1} a_{2} \ldots a_{n-1}$ folgt z.B.: Eine Dreiecksfolge ist genau dann streng n-periodisch, wenn die Anfangswinkel $\alpha, \beta, \gamma$ die Form $\alpha=\frac{p}{2^{n}-1} \pi, \beta=\frac{q}{2^{n}-1} \pi$, 
$\gamma=\frac{r}{2^{n}-1} \pi$ oder die Form $\alpha=\frac{p}{2^{n}+1} \pi, \beta=\frac{q}{2^{n}+1} \pi, \quad \gamma=\frac{r}{2^{n}+1} \pi$ mit ganzen $p, q, r$ haben. Anfängliche Verzögerung von $d$ Schritten wird mit dem Nenner $2^{d}\left(2^{n} \pm 1\right)$ erreicht. Die analogen Ausdrücke für $n$-periodische, aber nicht streng $n$-periodische Folgen lassen sich zwar ebenso ohne Mühe gewinnen, jedoch haben sie eine etwas unübersichtlichere Gestalt (diesbezüglich sei auf [14] verwiesen).

Mit Satz 4.6 sehen wir, dass es unendlich viele periodische Folgen mit paarweise nicht ähnlichen Ausgangsdreiecken gibt. Für ein festes $n$ sind es aber nur endlich viele, und zwar insgesamt $2^{n}\left(2^{n}-1\right) n$-periodische und davon $\left(4^{n}+2\right) / 6$ streng $n$-periodische; das bekommt man z.B. mit Burnsides Lemma nach einer längeren Rechnung (in [14] ist u.a. eine andere nur elementare kombinatorische Überlegungen benutzende Herleitung der ersten Formel zu finden). 1-periodische Folgen sind die mit den Anfangswinkeln $\left(\frac{\pi}{3}, \frac{\pi}{3}, \frac{\pi}{3}\right)$ und $\left(\frac{\pi}{7}, \frac{2 \pi}{7}, \frac{4 \pi}{7}\right)$ (die erste davon streng periodisch); 2-periodische Folgen sind die mit den Anfangswinkeln $\left(\frac{\pi}{3}, \frac{\pi}{3}, \frac{\pi}{3}\right),\left(\frac{\pi}{5}, \frac{\pi}{5}, \frac{3 \pi}{5}\right),\left(\frac{\pi}{5}, \frac{2 \pi}{5}, \frac{2 \pi}{5}\right),\left(\frac{\pi}{7}, \frac{2 \pi}{7}, \frac{4 \pi}{7}\right),\left(\frac{\pi}{13}, \frac{3 \pi}{13}, \frac{9 \pi}{13}\right)$, $\left(\frac{2 \pi}{13}, \frac{5 \pi}{13}, \frac{6 \pi}{13}\right),\left(\frac{\pi}{3}, \frac{2 \pi}{15}, \frac{8 \pi}{15}\right),\left(\frac{2 \pi}{3}, \frac{\pi}{15}, \frac{4 \pi}{15}\right),\left(\frac{\pi}{5}, \frac{\pi}{15}, \frac{11 \pi}{15}\right),\left(\frac{2 \pi}{5}, \frac{2 \pi}{15}, \frac{7 \pi}{15}\right),\left(\frac{\pi}{21}, \frac{4 \pi}{21}, \frac{16 \pi}{21}\right)$ und $\left(\frac{2 \pi}{21}, \frac{8 \pi}{21}, \frac{11 \pi}{21}\right)$ (die ersten drei davon streng periodisch).

Schließlich lassen sich zu drei vorgegebenen Winkeln $\alpha_{0}, \beta_{0}, \gamma_{0}$ mit $0 \leq \alpha_{0}, \beta_{0}, \gamma_{0} \leq \pi$, $\alpha_{0}+\beta_{0}+\gamma_{0}=\pi$ stets solche periodischen Folgen finden, deren Anfangswinkel sich von diesen beliebig wenig unterscheiden, d.h. die periodischen Folgen liegen „dicht“. Für $\left|\alpha-\alpha_{0}\right|,\left|\beta-\beta_{0}\right|,\left|\gamma-\gamma_{0}\right|<\frac{\pi}{2^{n}}$ ist das mit Periode $n$ zu erreichen: Die ersten $n$ Binärstellen der Verhältnisgrößen setze man gleich denen von $\frac{\alpha}{\pi}, \frac{\beta}{\pi}, \frac{\gamma}{\pi}$ und die übrigen gemäß einer Variante aus Satz 4.6, mit der keine Periode $\overline{0}$ oder $\overline{1}$ entsteht; ggf. schreibe man $\frac{\alpha}{\pi}, \frac{\beta}{\pi}$ bzw. $\frac{\gamma}{\pi}$ zuvor mit Periode $\overline{1}$.

Ich möchte mich bei Frau Prof. Dr. Irmtraud Stephani (FSU Jena), die mir bei der Erstellung dieser Arbeit stets hilfreich zur Seite stand, sowie bei Herrn Prof. Dr. Hans-Jürgen Schmeißer (FSU Jena) für die wertvollen Kommentare und Ratschläge herzlich bedanken.

\section{Literatur}

[1] Alexander, J.C.: The symbolic dynamics of the sequence of pedal triangles. Math. Mag. 66 (1993), 147158.

[2] Anglesio, J.: A Nowhere-Differentiable Function from Geometry: 10790. Amer. Math. Monthly 108 (2001), 568

[3] Arslanagić, Š.: Die Abstände der besonderen Punkte im Dreieck. Die Wurzel 5 (1999), 90-97 und 6 (1999), 114-120.

[4] Bankoff, L.; Garfunkel, J.: The heptagonal triangle. Math. Mag. 46 (1973), 7-19.

[5] Conway, J.H.; Lawrence, E.; Parish, J.; Ehrmann, J.-P.: The orthic limit. http://tech.groups.yahoo.com/group/Hyacinthos/message/5638 und $5640,5641,5647,5655$.

[6] Heurteaux, Y.: Weierstrass functions in Zygmund's class. Proc. Amer. Math. Soc. 133 (2005), 2711-2720.

[7] Hobson, E.W.: A treatise on plane trigonometry. Cambridge University Press, 1891, 194-200.

[8] Kingston, J.G.; Synge, J.L.: The sequence of pedal triangles. Amer. Math. Monthly 95 (1988), 609-620. 
[9] Lax, P.D.: The ergodic character of sequences of pedal triangles. Amer. Math. Monthly 97 (1990), 377381

[10] Sloane, N.J.A.: The On-Line Encyclopedia of Integer Sequences. http: //www.research.att.com/ njas/sequences

[11] Taylor, D.G.: Successive Pedal Triangles. Math. Gazette 30 (1946), 11-13.

[12] Tuckey, C.O.: Angles of pedal triangles. Math. Gazette 17 (1933), 48-49.

[13] Ungar, P.: Mixing property of the pedal mapping. Amer. Math. Monthly 97 (1990), 898-900.

[14] Vályi, J.: Über die Fußpunktdreiecke. Monatshefte für Mathematik und Physik 14 (1903), 243-253.

Evgeny Strekalovskiy

Friedensgasse 16

D-99423 Weimar, Deutschland

e-mail: evgeny.strekalovskiy@gmx.de 